IC049932K Supporting Information

\title{
Structure and Dynamics of Dinuclear Zirconium(IV) Complexes
}

Weiqing Zhong, John A. Parkinson, Simon Parsons, Iain D. H. Oswald,

Robert A. Coxall and Peter J. Sadler

Table S1

Figures S1-S5 
Table S1 Selected Bond Lengths $(\AA)$ and Angles $\left(^{\circ}\right)$ for $\mathrm{K}_{2}\left[\mathrm{Zr}_{2}(\mathrm{dhpta})_{2}\right] \cdot 5 \mathrm{H}_{2} \mathrm{O}$

(2.5 $\left.\mathrm{H}_{2} \mathrm{O}\right), \mathrm{Na}_{2} \mathrm{Zr}_{2} \mathrm{C}_{22} \mathrm{H}_{26} \mathrm{~N}_{4} \mathrm{O}_{18} \cdot 7 \mathrm{H}_{2} \mathrm{O} \cdot \mathrm{C}_{2} \mathrm{H}_{5} \mathrm{OH}\left(\mathbf{3} \cdot 7 \mathrm{H}_{2} \mathrm{O} \cdot \mathrm{C}_{2} \mathrm{H}_{5} \mathrm{OH}\right)$, and

$\mathrm{Cs}_{2} \mathrm{Zr}_{2} \mathrm{C}_{22} \mathrm{H}_{26} \mathrm{~N}_{4} \mathrm{O}_{18} \cdot \mathrm{H}_{5} \mathrm{O}_{2} \cdot \mathrm{Cl} \cdot 4 \mathrm{H}_{2} \mathrm{O}\left(4 \cdot \mathrm{H}_{5} \mathrm{O}_{2} \cdot \mathrm{Cl} \cdot 4 \mathrm{H}_{2} \mathrm{O}\right)$

\begin{tabular}{llll}
\hline \multicolumn{4}{c}{ Complex $2 \cdot 5 \mathrm{H}_{2} \mathrm{O}^{\mathrm{a}}$} \\
\hline \multicolumn{5}{c}{ bond length } \\
\hline $\mathrm{K}(1)-\mathrm{K}(2)$ & $3.9222(38)$ & $\mathrm{K}(2)-\mathrm{O}(7 \mathrm{BA})$ & $2.811(3)$ \\
$\mathrm{K}(1)-\mathrm{O}(4 \mathrm{~A})$ & $2.735(2)$ & $\mathrm{K}(2)-\mathrm{O}(4 \mathrm{BA})$ & $2.880(3)$ \\
$\mathrm{K}(1)-\mathrm{O}(1 \mathrm{BA})$ & $2.765(2)$ & $\mathrm{K}(2)-\mathrm{O}(2 \mathrm{~W})$ & $2.763(3)$ \\
$\mathrm{K}(1)-\mathrm{O}(3 \mathrm{AA})$ & $2.862(2)$ & $\mathrm{K}(2)-\mathrm{O}(3 \mathrm{WA})$ & $2.765(3)$ \\
$\mathrm{K}(1)-\mathrm{O}(7 \mathrm{~A})$ & $2.991(2)$ & $\mathrm{K}(2)-\mathrm{O}(5 \mathrm{WB})$ & $3.109(5)$ \\
$\mathrm{K}(1)-\mathrm{O}(1 \mathrm{~W})$ & $2.709(3)$ & \multicolumn{2}{c}{ bond angle } \\
$\mathrm{K}(1)-\mathrm{O}(2 \mathrm{~W})$ & $2.810(2)$ & $\mathrm{K}(1)-\mathrm{O}(4 \mathrm{~A})-\mathrm{K}(2)$ & $92.74(7)$ \\
$\mathrm{K}(1)-\mathrm{O}(1 \mathrm{WA})$ & $2.915(3)$ & $\mathrm{K}(1)-\mathrm{O}(2 \mathrm{~W})-\mathrm{K}(2)$ & $89.47(7)$ \\
$\mathrm{K}(1)-\mathrm{O}(3 \mathrm{WA})$ & $3.000(2)$ & $\mathrm{K}(1)-\mathrm{O}(3 \mathrm{~W})-\mathrm{K}(2)$ & $85.64(7)$ \\
$\mathrm{K}(2)-\mathrm{O}(4 \mathrm{~A})$ & $2.684(2)$ & \multicolumn{2}{c}{} \\
\hline
\end{tabular}

Complex 3.7 $\mathrm{H}_{2} \mathrm{O} \cdot \mathrm{C}_{2} \mathrm{H}_{5} \mathrm{OH}^{\mathrm{a}}$

\begin{tabular}{|c|c|c|c|}
\hline \multicolumn{4}{|c|}{ bond length } \\
\hline $\mathrm{Na}(1)-\mathrm{Na}(2)$ & $3.741(10)$ & $\mathrm{Na}(2)-\mathrm{O}(92 \mathrm{~A})$ & $2.448(12)$ \\
\hline $\mathrm{Na}(1)-\mathrm{O}(121)$ & $2.394(2)$ & $\mathrm{Na}(2)-\mathrm{O}(12 \mathrm{~A})$ & $2.486(10)$ \\
\hline $\mathrm{Na}(1)-\mathrm{O}(111)$ & $2.717(2)$ & $\mathrm{Na}(2)-\mathrm{O}(2 \mathrm{~W})$ & $2.106(8)$ \\
\hline $\mathrm{Na}(1)-\mathrm{O}(92 \mathrm{~A})$ & $2.517(2)$ & $\mathrm{Na}(2)-\mathrm{O}(7 \mathrm{~W})$ & $2.384(12)$ \\
\hline $\mathrm{Na}(1)-\mathrm{O}(5 \mathrm{~W})$ & $2.250(6)$ & $\mathrm{Na}(2)-\mathrm{O}(5 \mathrm{~W})$ & $2.900(9)$ \\
\hline $\mathrm{Na}(1)-\mathrm{O}(6 \mathrm{~W})$ & $2.373(3)$ & & \\
\hline $\mathrm{Na}(1)-\mathrm{O}(6 \mathrm{WA})$ & $2.459(3)$ & bond a & igle \\
\hline $\mathrm{Na}(1)-\mathrm{O}(91 \mathrm{~A})$ & $3.005(2)$ & $\mathrm{Na}(1)-\mathrm{O}(5 \mathrm{~W})-\mathrm{Na}(2)$ & $92.3(3)$ \\
\hline $\mathrm{Na}(2)-\mathrm{O}(122)$ & $2.241(12)$ & $\mathrm{Na}(1)-\mathrm{O}(92 \mathrm{~A})-\mathrm{Na}(2)$ & $97.8(3)$ \\
\hline \multicolumn{4}{|c|}{ Complex $4 \cdot \mathrm{H}_{5} \mathrm{O}_{2} \cdot \mathrm{Cl} \cdot 4 \mathrm{H}_{2} \mathrm{O}^{\mathrm{a}}$} \\
\hline \multicolumn{4}{|c|}{ bond length } \\
\hline $\operatorname{Cs}(1)-\operatorname{Cs}(2)$ & $4.682(5)$ & $\mathrm{Cs}(2)-\mathrm{O}(202)$ & $3.066(3)$ \\
\hline $\mathrm{Cs}(1)-\mathrm{O}(222)$ & $3.049(3)$ & $\mathrm{Cs}(2)-\mathrm{O}(12 \mathrm{C})$ & $3.534(4)$ \\
\hline $\mathrm{Cs}(1)-\mathrm{O}(18 \mathrm{~B})$ & $3.241(3)$ & $\mathrm{Cs}(2)-\mathrm{O}(222)$ & $3.614(3)$ \\
\hline $\mathrm{Cs}(1)-\mathrm{O}(92 \mathrm{~A})$ & $3.416(3)$ & $\mathrm{Cs}(2)-\mathrm{O}(221)$ & $3.645(3)$ \\
\hline $\mathrm{Cs}(1)-\mathrm{O}(12 \mathrm{~B})$ & $3.421(3)$ & $\mathrm{Cs}(2)-\mathrm{O}(2 \mathrm{~W})$ & $3.275(4)$ \\
\hline $\mathrm{Cs}(1)-\mathrm{O}(18 \mathrm{~A})$ & $3.441(3)$ & $\mathrm{Cs}(2)-\mathrm{O}(1 \mathrm{WA})$ & $3.287(4)$ \\
\hline $\mathrm{Cs}(1)-\mathrm{O}(12 \mathrm{~A})$ & $3.482(3)$ & $\mathrm{Cs}(2)-\mathrm{Cl}(1)$ & $3.4174(12)$ \\
\hline $\mathrm{Cs}(1)-\mathrm{O}(72 \mathrm{~A})$ & $3.625(3)$ & $\mathrm{Cs}(2)-\mathrm{O}(201)$ & $3.778(3)$ \\
\hline $\mathrm{Cs}(1)-\mathrm{O}(1 \mathrm{~W})$ & $3.086(4)$ & \multicolumn{2}{|c|}{ bond angle } \\
\hline $\mathrm{Cs}(1)-\mathrm{Cl}(1 \mathrm{~A})$ & $3.3834(12)$ & $\mathrm{Cs}(1)-\mathrm{O}(222)-\mathrm{Cs}(2)$ & $88.85(8)$ \\
\hline $\mathrm{Cs}(2)-\mathrm{O}(18 \mathrm{~B})$ & $3.029(3)$ & $\mathrm{Cs}(1)-\mathrm{O}(18 \mathrm{~B})-\mathrm{Cs}(2)$ & $96.53(8)$ \\
\hline
\end{tabular}

${ }^{\mathrm{a}}$ Estimated standard deviations are given in parentheses. See Figs. 1 and $\mathrm{S} 1$ for atom labels. 


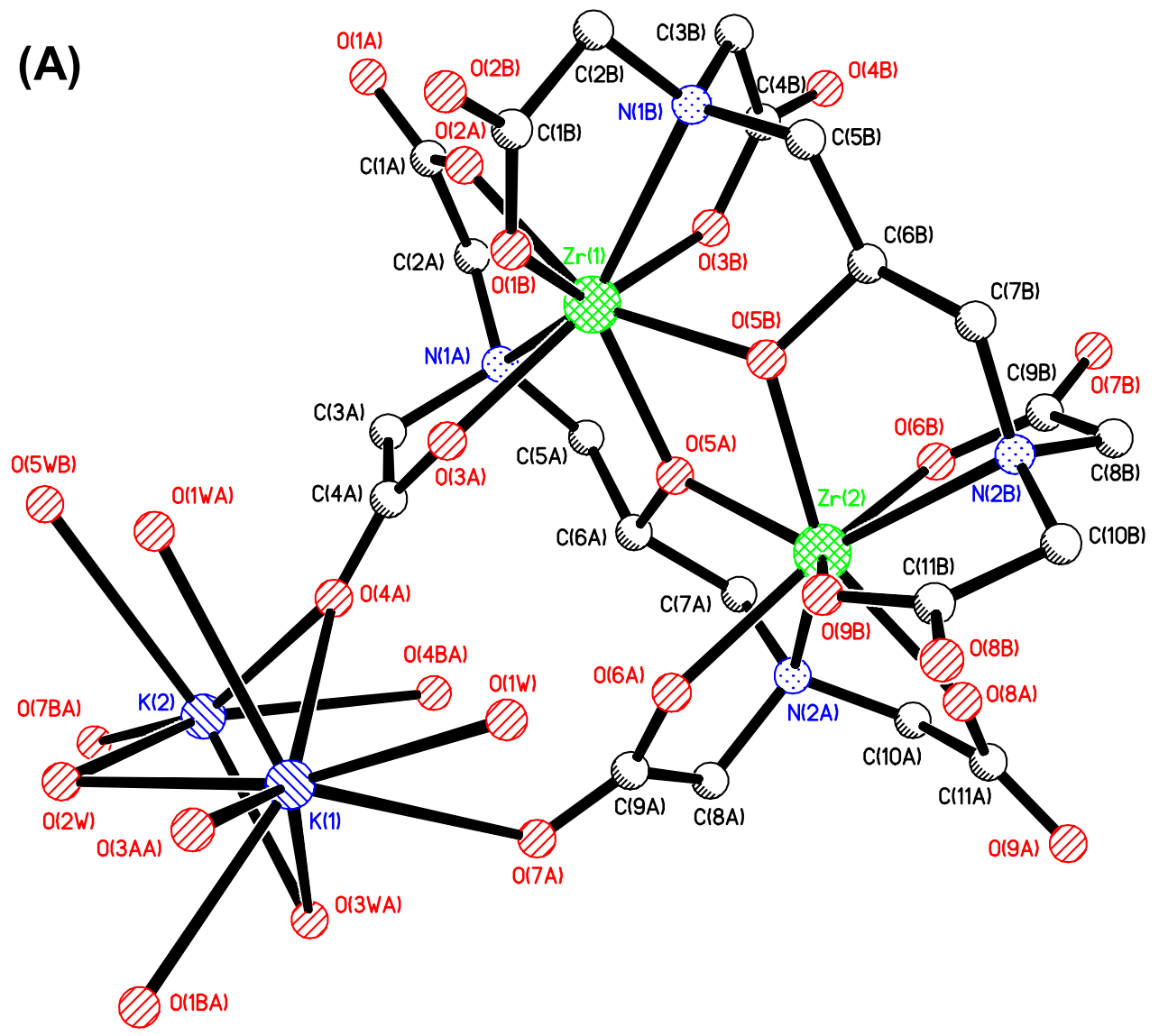

(B)

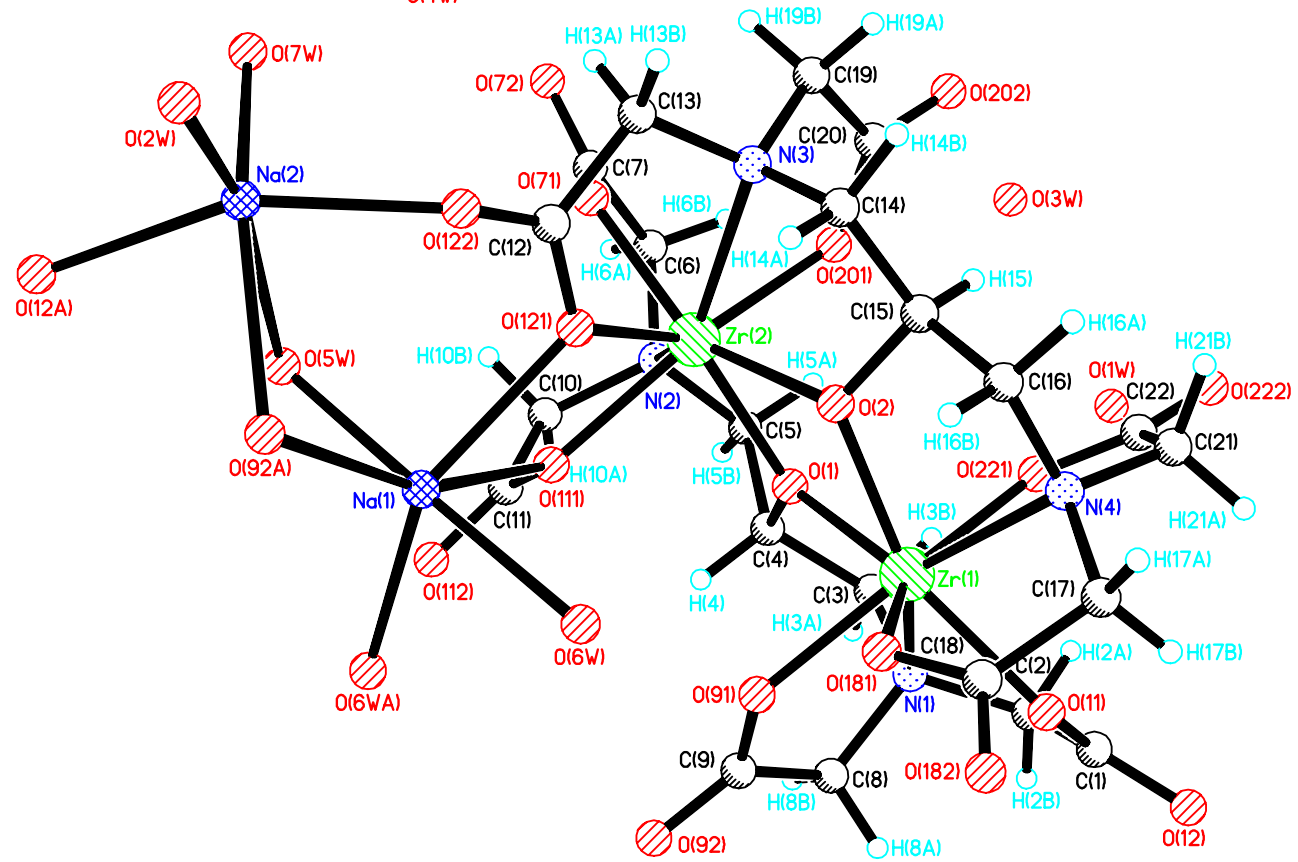




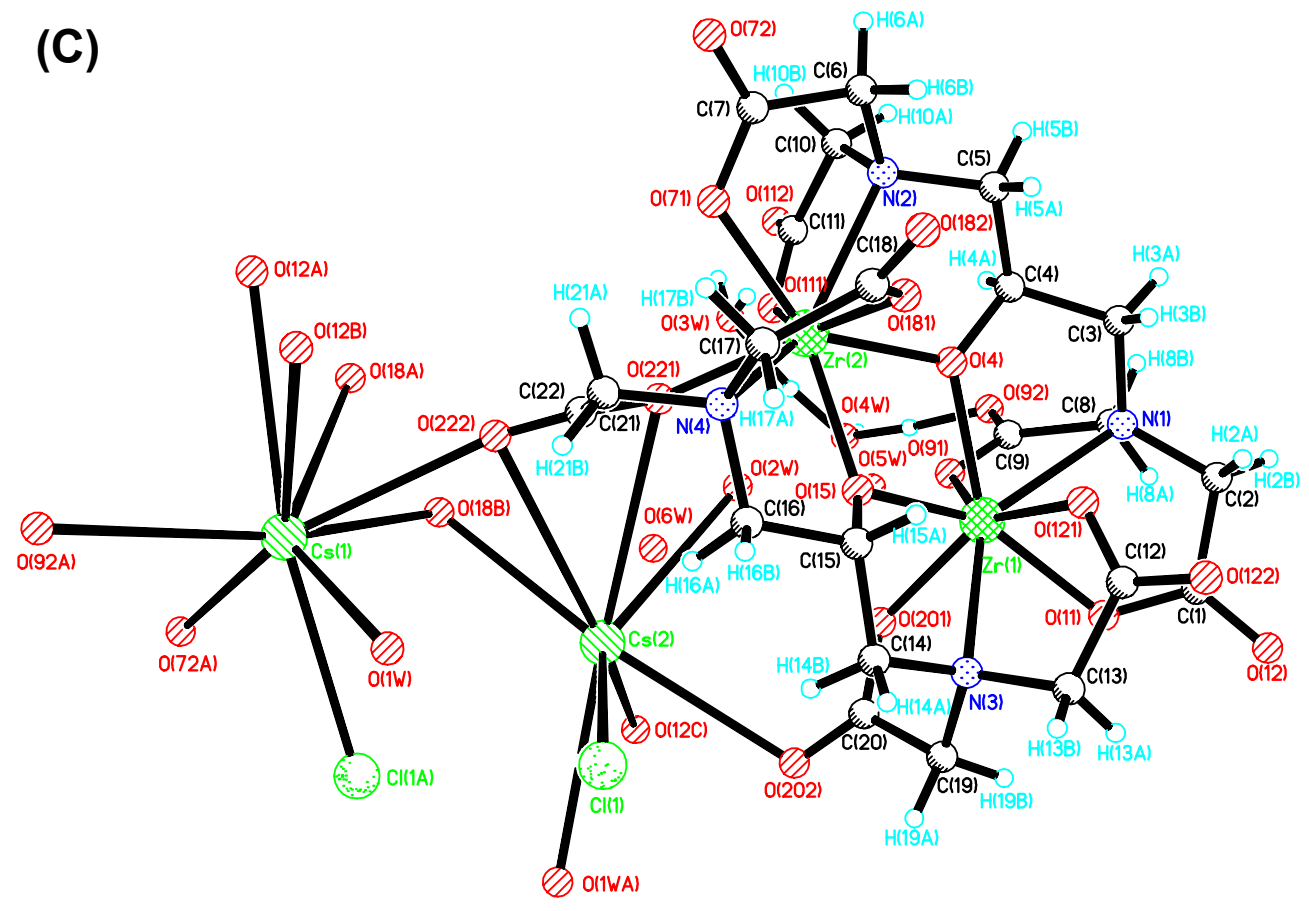

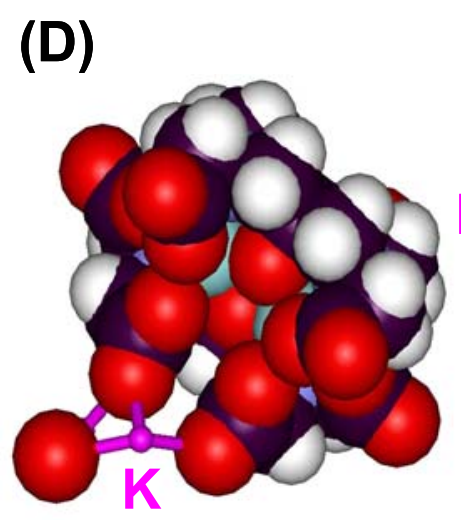

2

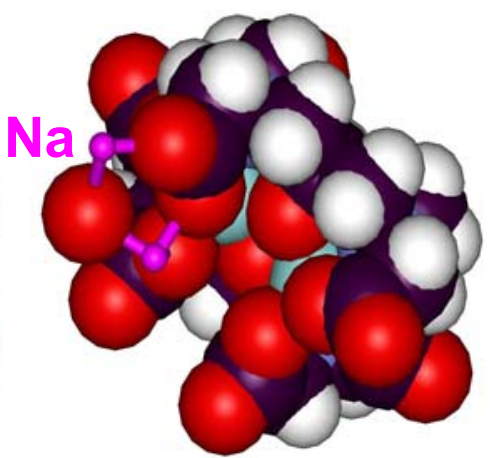

3

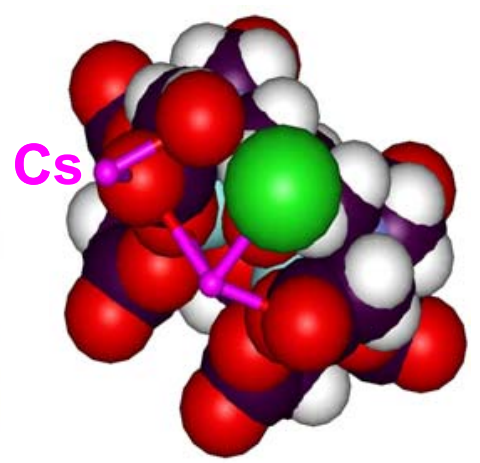

4

Figure S1. Diagrams showing the coordination of the alkali metal ions to the dinuclear anion $\left[\mathrm{Zr}_{2}(\text { dhpta })_{2}\right]^{2-}$. (A) $\mathrm{K}_{2}\left[\mathrm{Zr}_{2}(\text { dhpta })_{2}\right] \cdot 5 \mathrm{H}_{2} \mathrm{O} \quad\left(\mathbf{2} \cdot 5 \mathrm{H}_{2} \mathrm{O}\right)$, (B) $\left.\mathrm{Na}_{2}\left[\mathrm{Zr}_{2} \text { (dhpta) }\right)_{2}\right] \cdot 7 \mathrm{H}_{2} \mathrm{O}\left(\mathbf{3} \cdot 7 \mathrm{H}_{2} \mathrm{O}\right)$, and (C) $\mathrm{Cs}_{2}\left[\mathrm{Zr}_{2}(\text { dhpta })_{2}\right] \cdot \mathrm{H}_{5} \mathrm{O}_{2} \cdot \mathrm{Cl} \cdot 4 \mathrm{H}_{2} \mathrm{O} \quad\left(\mathbf{4} \cdot \mathrm{H}_{5} \mathrm{O}_{2}\right.$ $\cdot \mathrm{Cl} \cdot 4 \mathrm{H}_{2} \mathrm{O}$ ). (D) Space-filling models of the dinuclear anions in complexes $\mathbf{2}, \mathbf{3}$ and $\mathbf{4}$, with bound alkali metal ions as purple balls. The water oxygen atoms bridging the $\mathrm{K}(\mathrm{I})$ ions in $\mathbf{2}$ and $\mathrm{Na}(\mathrm{I})$ ions in $\mathbf{3}$, and $\mathrm{Cl}^{-}$(green) bound to $\mathrm{Cs}(\mathrm{I})$ in $\mathbf{4}$ are also shown. 


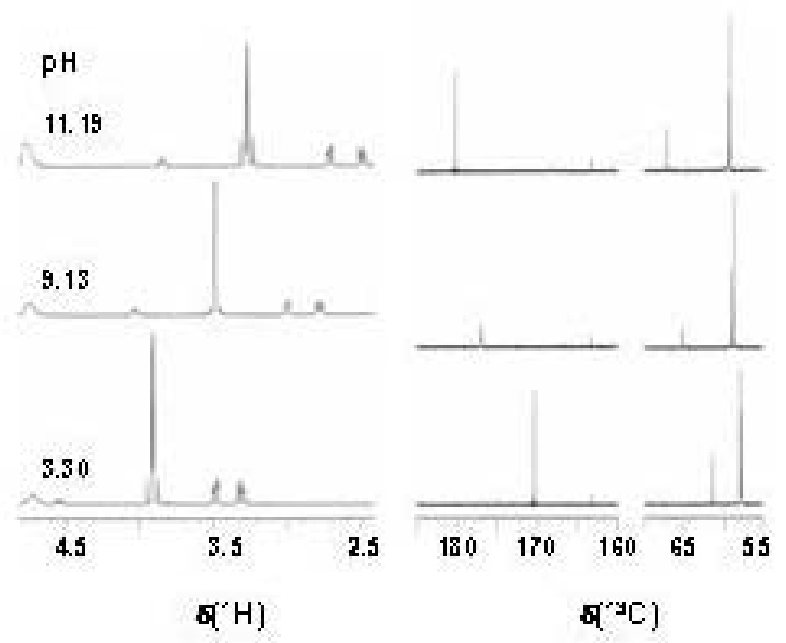

Figure S2. Dependence of the ${ }^{1} \mathrm{H}$ (left) and ${ }^{13} \mathrm{C}-\left\{{ }^{1} \mathrm{H}\right\}$ (right) NMR spectra of the ligand $\mathrm{H}_{5}$ dhpta on $\mathrm{pH}$. The peak at $163.21 \mathrm{ppm}$ is for the external reference $\mathrm{NaH}\left[{ }^{13} \mathrm{C}\right] \mathrm{O}_{3}$. 


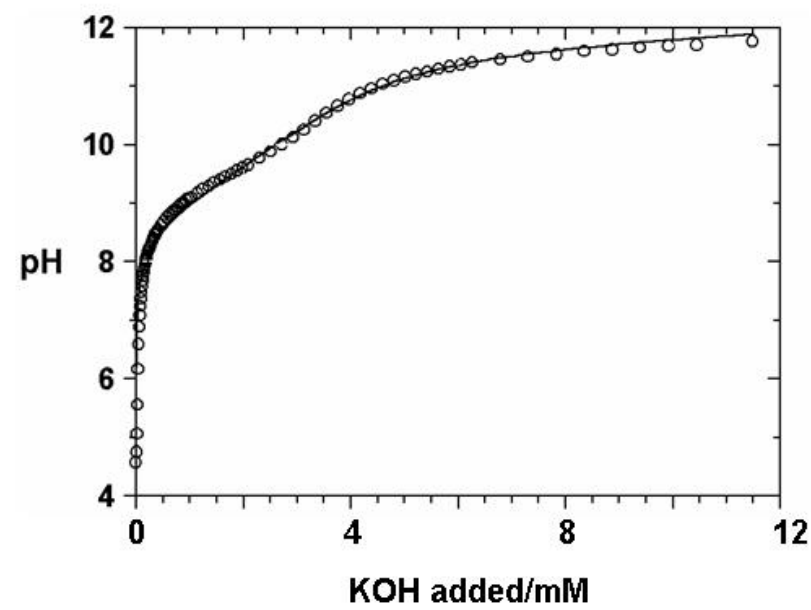

Figure S3. pH titration of $\mathrm{K}_{2} \mathrm{Zr}_{2}(\text { dhpta })_{2}(5.94 \mathrm{mM} \mathrm{Zr})$ with $0.2028 \mathrm{M} \mathrm{KOH}$ at 293 $\mathrm{K}$. The line represents a computer fit giving $\mathrm{pK}_{\mathrm{a}}$ values of $\mathrm{pK}_{\mathrm{a} 1}=9.02 \pm 0.09$ and $\mathrm{pK}_{\mathrm{a} 2}$ $=10.02 \pm 0.05$. 


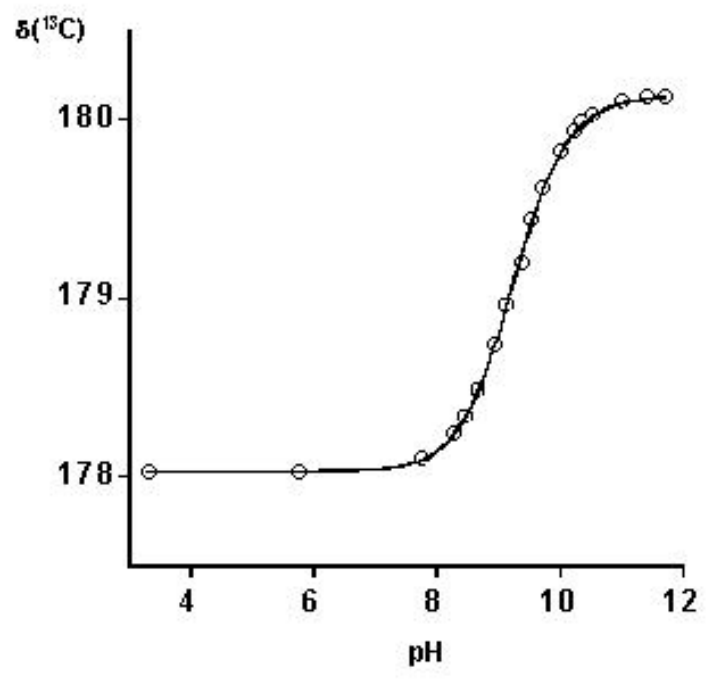

Figure S4. Dependence of the ${ }^{13} \mathrm{C}$ NMR chemical shift of carboxyl peak $4 \mathbf{A}$ of complex 2 (for labels see Fig. 3) on pH at $298 \mathrm{~K}$ in 9:1 $\mathrm{H}_{2} \mathrm{O}: \mathrm{D}_{2} \mathrm{O}$. The line represents a computer fit giving $\mathrm{pK}_{\mathrm{a}}$ values of $\mathrm{pK}_{\mathrm{a} 1}=9.14 \pm 0.29$ and $\mathrm{pK}_{\mathrm{a} 2}=10.04 \pm 0.52$. 


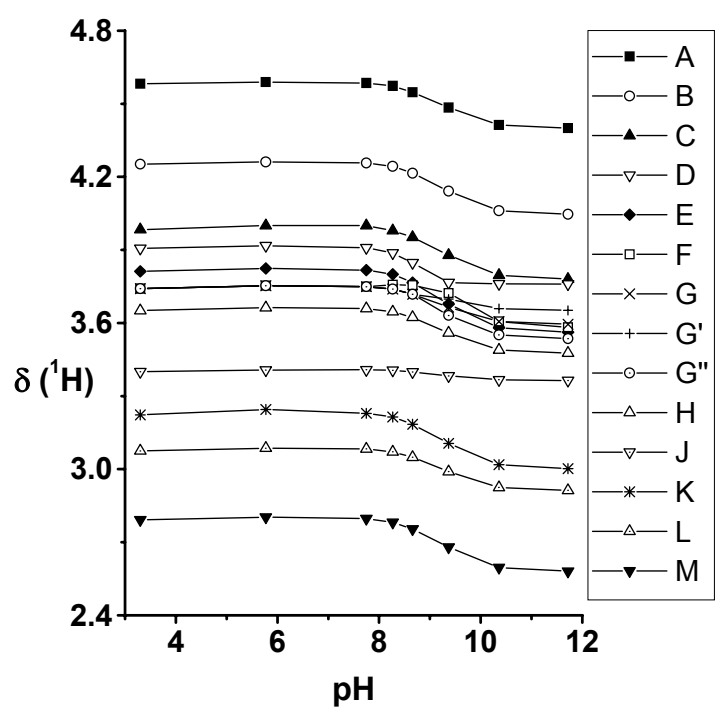

Figure S5. Dependence of the ${ }^{1} \mathrm{H}$ chemical shifts for complex 2 on $\mathrm{pH}$ at $298 \mathrm{~K}$. Assignments of peaks $\mathbf{A}-\mathbf{M}$ are shown in Figure 3. 